\title{
CHIRI WAYRITA: LA VOZ DE LOS SIN VOZ. UNA EXPERIENCIA DE EDUCACIÓN TRANSFORMADORA PARA LOS NIÑOS Y NIÑAS DEL CERRO RICO (BOLIVIA)
}

\section{Chiri Wayrita: The voice of the voiceless. A transformative education experience for the children of Cerro Rico (Bolivia)}

\author{
M. ${ }^{a}$ Dolores Fernández Malanda \\ Universidad de Burgos \\ Correo-e: fermal@ubu.es \\ Mara GARcía Rodríguez \\ Universidad de Burgos \\ Correo-e: maragr@ubu.es \\ Recibido: I3 de noviembre de 2019 \\ Envío a informantes: 22 de noviembre de 2019 \\ Aceptación definitiva: I4 de mayo de 2020
}

Resumen: Desde hace una década, la Universidad de Burgos, a través del Vicerrectorado de Internacionalización y Cooperación y el Centro de Cooperación y Acción Solidaria, apuesta por proyectos educativos y de salud en Bolivia, concretamente en el Departamento de Potosí. A lo largo de estos años, estudiantes y profesores de diferentes titulaciones hemos participado de una experiencia que ha cambiado nuestra visión del mundo al acercarnos a culturas que sobreviven al individualismo, la cosificación y la despersonalización, pilares del capitalismo neoliberal salvaje, empoderando y fortaleciendo el sentimiento de comunidad. Convivir con los pueblos andinos, quechuas y aymaras; participar de sus modelos de educación propia en escuelas tan complejas y hermosas como Robertito, edificada en el corazón de una de las minas más terribles del planeta, el Sumaq Orcko, nos ha hecho adquirir una nueva y enriquecedora visión del mundo a partir de una cosmovisión para nosotros reveladora por los principios de solidaridad y reciprocidad sobre los que se ha construido (Fernández Malanda, D. en Cifuentes García y Gómez Campelo, 20I6: I2I). 
CHIRI WAYRITA: LA VOZ DE LOS SIN VOZ. UNA EXPERIENCIA DE EDUCACIÓN TRANSFORMADORA PARA LOS NIÑOS Y NIÑAS DEL CERRO RICO (BOLIVIA) M. ${ }^{a}$ DOLORES FERNÁNDEZ MALANDA Y MARA GARCÍA RODRÍGUEZ

Palabras clave: niños, niñas y adolescentes trabajadores; explotación infantil; mina; Escuela Robertito; protagonismo infantil.

AвsтRAct: For a decade, the University of Burgos, through the Vice-Rectorate for Internationalization and Cooperation, and the Center for Cooperation and Solidarity Action, is committed to a series of educational and health projects in Bolivia, specifically in the Department of Potosí. Throughout these years, students and teachers of different degrees have participated in an experience that has changed our worldview by approaching cultures that survive individualism, reification and depersonalization, pillars of wild neoliberal capitalism, empowering and strengthening the feeling of community. Living with the Andean, Quechua and Aymara peoples, participating in their own education models in schools as complex and beautiful as Robertito, built in the heart of one of the most terrible mines on the planet, the Sumaq Orcko, has made us acquire a new and enriching vision of the world from a worldview revealing to us by the principles of solidarity and reciprocity upon which it has been built (Fernández Malanda, D. in Cifuentes García and Gómez Campelo, 20I6: I2I).

KEY wORDs: working children and adolescents; child exploitation; mine; Robertito School; child protagonism.

\section{Introducción}

$\mathrm{N}$

O PODEMOS HABLAR DE ROBERTITO sin hacer un recorrido necesario por el país, la ciudad y la situación de los niños, niñas y adolescentes que acoge en sus aulas.

Bolivia, considerado uno de los Estados más pobres de América Latina, es un país marcado tanto por la gran heterogeneidad geográfica y étnica como por los elevados índices de desigualdad económica y social.

Sobre su heterogeneidad cultural, tal como recoge la Constitución Política del Estado Plurinacional, conviven 36 naciones indígenas y originarias, y 34 lenguas oficiales reconocidas en 20I2. Según los datos del censo más fiable hasta el momento, el censo del 20II, la población indígena representa el 62\% del total (Io.671.200 millones de habitantes según el censo de 2013), en su mayoría integrada por quechuas, aymaras y guaraníes, conviviendo con sectores mestizos y criollos (García Rodríguez, 2015).

En cuanto a la desigualdad económica, el 63\% de los bolivianos está por debajo de la línea de pobreza, es decir, no tiene ingresos suficientes para satisfacer sus necesidades básicas; el $37 \%$ no puede cubrir ni siquiera sus necesidades alimenticias. Esta asimetría tiene su correspondencia étnica, dado que en el área rural -donde se da la mayor concentración de población indígena- es donde la pobreza puede llegar hasta el 8I,7\% como es el caso del departamento de Potosí. El binomio indígena-pobreza es un complejo histórico, como lo fueron también las violaciones de Derechos Humanos fundamentales (Citarella Menardi, 20Io: I4).

Dentro de este contexto de grandes contrastes destaca Potosí, la segunda ciudad más alta del mundo (posee una altura media de $3900 \mathrm{msnm}$ ). Conocida por su actividad minera, que se remonta a la época precolombina, la urbe se expande a los pies del Cerro 
CHIRI WAYRITA: LA VOZ DE LOS SIN VOZ. UNA EXPERIENCIA DE EDUCACIÓN TRANSFORMADORA PARA LOS NIÑOS Y NIÑAS DEL CERRO RICO (BOLIVIA) M. ${ }^{a}$ DOLORES FERNÁNDEZ MALANDA Y MARA GARCÍA RODRÍGUEZ

Rico. Las famosas minas de plata explotadas durante los siglos de la Colonia han dado paso a la extracción de otros minerales, como el estaño y el zinc. La dureza de la vida en esta ciudad se refleja en la situación de las familias potosinas, la mayoría de las cuales viven bajo el umbral de la pobreza y la extrema pobreza, trabajando en condiciones altamente precarias.

En esta realidad, son los y las NAT (niños, niñas y adolescentes trabajadores) los que de manera más directa ven vulnerados sus derechos básicos. Obligados a insertarse en el mundo laboral desde temprana edad, en muchas ocasiones su trabajo se realiza bajo condiciones de explotación. Esta situación impide el acceso a la formación, educación y especialización de los/as NAT.

Pese a que la legislación boliviana establece la edad mínima para el trabajo en io años, son muchos los niños y niñas menores de io años que debido a su situación familiar desempeñan una actividad remunerada para garantizar su acceso a la educación, alimentación y necesidades básicas, incluyendo el apoyo a sus familias. Las dificultades para el acceso a derechos básicos como la salud o la educación, así como los beneficios obtenidos de poseer un trabajo regulado, si bien pueden justificar la excepcionalidad del trabajo infantil en determinadas circunstancias, no han de ser una excusa para no proporcionar tales derechos básicos (Constitución Política del Estado Plurinacional de Bolivia, 2009).

\section{Contexto}

El Departamento de Potosí cuenta con 16 provincias y 38 municipios, su población alcanza a 825.000 habitantes (según el censo de 2012 ), siendo un $66 \%$ población rural y el restante $34 \%$ población urbana. En cuanto a la pobreza Potosí ocupa los primeros lugares en la escala de la pobreza con un promedio de 1,5 dólares de ingreso día por familia.

La ciudad de Potosí se sitúa al sur de Bolivia y es la capital del departamento. Se ubica a unos 3900 metros de altitud, a los pies de la legendaria montaña del Cerro Rico, en la que se localizó la mina de plata más grande del mundo a mediados del siglo XVI, iniciándose un «cataclísmico» proceso de explotación que en 500 años acabó con la vida de más de 8 millones de seres humanos (Bascopé, 2013). Cuenta con una población media de unos 250.000 habitantes y fue declarada Patrimonio de la Humanidad por la Unesco en 1987 , dado su gran patrimonio arquitectónico que se compone de numerosas construcciones coloniales (catedral, Casa de la Moneda, la Universidad Tomás Frías o la Torre de la Compañía), y el enorme valor histórico del Cerro.

Potosí nació y prosperó gracias a la minería, pero las reservas de plata que la hicieron internacional están casi agotadas. En la actualidad, la actividad minera se centra en el estaño y el zinc, en proyectos de concentración de minerales de baja ley (sobre todo desechos de plata acumulados desde épocas coloniales) y derivados.

La agricultura de la región es de tipo altiplánico. Sobresalen las producciones de papa en sus diferentes variedades; quinua real, cuya demanda excede grandemente la oferta; algunas especies de maíz, y productos alimenticios varios destinados, en general, a consumo y mercado local. La industria es escasa, solamente existen algunas empresas medianas en el rubro de la cerveza o el procesado de alimentos. 

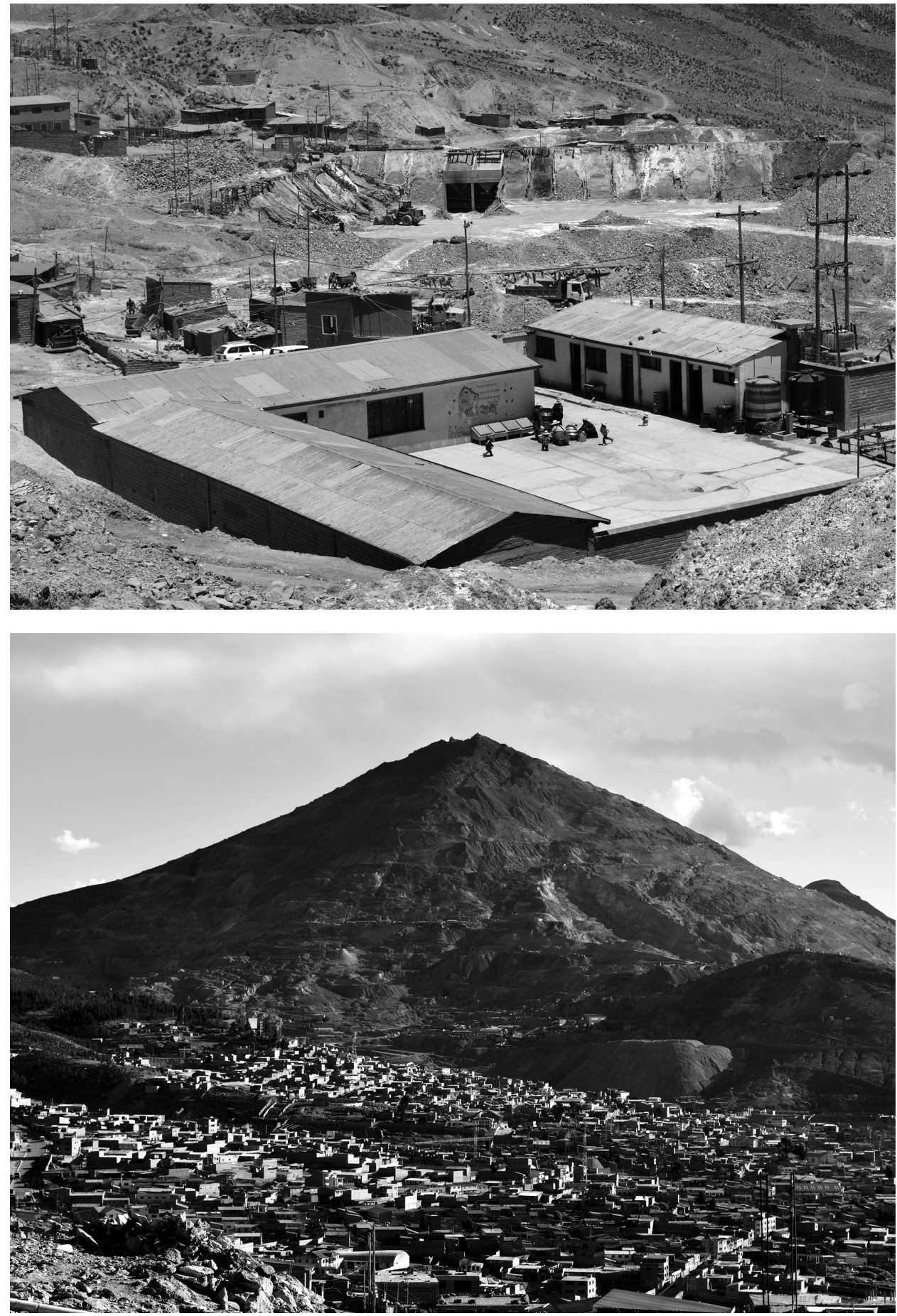

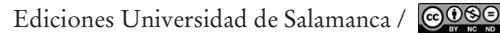

Aula, 26, 2020, pp. 73-84 
CHIRI WAYRITA: LA VOZ DE LOS SIN VOZ. UNA EXPERIENCIA DE EDUCACIÓN TRANSFORMADORA PARA LOS NIÑOS Y NIÑAS DEL CERRO RICO (BOLIVIA) M. ${ }^{a}$ DOLORES FERNÁNDEZ MALANDA Y MARA GARCÍA RODRÍGUEZ

\section{I. La situación de los menores de Potosí}

En Bolivia, más de 800.000 niños, niñas y adolescentes de 5 a 17 años trabajan, según los datos del INE de 20II. Un porcentaje difícil de estimar «trabaja» en las minas, en las peores condiciones. De todas las minas del país, el Cerro Rico de Potosí es la más dura. En este contexto, el caso más extremo es el de los NAT que viven en el Cerro Rico, al pie de las minas, que son hijos/as de las palliri (mujeres guardas de las minas, en su mayoría viudas de mineros) y que sobreviven en condiciones infrahumanas.

Desde hace meses, muchas de ellas no perciben sus exiguos sueldos ya que la bajada del precio de los minerales que se extraen ha provocado una grave crisis del sector en el departamento de Potosí.

En el Cerro Rico no hay agua, y en la ciudad el agua no es potable, quien quiere beber agua potable debe comprarla embotellada. Las familias del Cerro beben el agua contaminada que dejan las empresas mineras muchas veces reutilizada por los habitantes del Cerro tras el uso de la misma para el lavado del mineral en los ingenios. Los niveles de plomo en los órganos de los habitantes de la montaña maldita, incluidos los niños y las niñas, son muy elevados, y en el resto de la ciudad los habitantes enferman de DEA (diarreas agudas) en un alto porcentaje. No hay luz, ni saneamientos. Miles de personas viven en chabolas de calamina de io metros cuadrados expuestas diariamente al polvo tóxico de cientos de minas que agujerean como un queso de gruyere al cerro Patrimonio de la Humanidad. En similar situación se encuentran las familias de mineros que viven en los barrios al pie del Cerro. El servicio más extendido en los hogares de los NAT del resto de la ciudad es la energía eléctrica (31,5\%) seguido por el agua $(30,8 \%)$ y el baño $(27,9 \%)$. Las actividades metalúrgicas también han tenido un fuerte impacto sobre las áreas verdes y de recreación en las zonas residenciales: en Potosí existen bajos índices de estos espacios, con las consiguientes consecuencias en la salud y el bienestar de la población. Esta situación, sumada a la falta de agua, impide también que las familias puedan cultivar sus propios alimentos, lo que incide de forma negativa en los altos niveles de desnutrición (PASOCAP, 20I4).

La realidad de pobreza del departamento ( $87 \%$ según los últimos datos de la CEPAL) hace que los hogares potosinos tengan que buscar diferentes medios para poder subsistir: por esta razón el trabajo infantil es común en las familias, en algunos casos los (as) niños/as trabajan desde los 5 años, ayudando a sus padres en diferentes labores o trabajos. En general, los ingresos de las familias de los NAT son muy bajos, pues la mayoría de los padres de familia trabajan en la mina como peón o ayudante, en talleres mecánicos como ayudantes o en la construcción como albañiles. En el caso de las mujeres, suelen trabajar como lavanderas, empleadas del hogar o en el comercio informal. En consecuencia, el nivel de ingreso familiar no excede los Izo dólares por mes que, para una familia de 8 a I2 integrantes, es insuficiente para satisfacer las necesidades más urgentes del hogar (CEPAL, 20I8).

En relación a la educación más del 40\% de los NAT no asiste a la escuela, y su edad no tiene correspondencia con el nivel o grado escolar en el que están matriculados en un alto porcentaje. Las razones son múltiples. Por un lado, afectan el escaso nivel económico familiar, el elevado número de hijos e hijas, la baja escolaridad de los padres, y también existen causas derivadas del propio sistema educativo como la limitación de profesores y servicios de apoyo en los centros, el elevado número de alumnos/as por 
aula, la falta de formación de los profesionales de la educación o la oferta educativa incompleta (PASOCAP, 2OI5).

Los/as NAT abandonan la escuela por la imposibilidad de conciliar el horario escolar con el laboral pese a que el Gobierno ha articulado medidas para que puedan acudir a los colegios creando en todos los centros públicos tres horarios de clase (mañana, tarde y noche). No obstante, en los últimos años, los incentivos económicos han contribuido a mejorar esta situación, sobre todo en la zona rural. Las personas sin recursos económicos raramente pueden asistir al médico, operarse o concluir tratamientos pese a la solidaridad de las familias y vecinos; no existe en Bolivia un seguro médico universal. Los NAT tampoco cuentan con atención en salud siendo las principales víctimas al estar sometidos a duras jornadas de trabajo en condiciones de extrema dureza física. Las necesidades de salud son generales, pero hay un colectivo muy castigados por razones obvias: los niños, niñas y adolescentes que trabajan en las minas. Aunque el Estado oferta un servicio de atención a través del Seguro Universal Materno Infantil (sumi), este solo cubre a menores de 5 años y mujeres embarazadas. La mayor parte de los NAT sufren de enfermedades infecciosas de tipo respiratorio, gastrointestinales, dermatológicas y problemas odontológicos en general.

Pese a las situaciones adversas que enfrentan, de acuerdo al diagnóstico elaborado por Pasocap, y recogido en el documento Línea de Base del Programa Derecho a la Protección contra las peores formas de trabajo de Bolivia: Proyecto «Más allá del sol», los NAT se encuentran muy motivados para salir adelante y poder aspirar a cambiar su realidad con esfuerzo y dedicación (PASOCAP, 20I4).

En cuanto al sector de trabajo de los NAT, dentro de la ciudad de Potosí, se han identificado diversidad de sectores. Los encuestados provienen de 38 sectores: albañilería, artesanía, atención de café Internet, ayudante de oficios varios, ayudante de cocina, labores de casa, perforista, barraquero, estibador, carpintería, chapista, chofer, cocinero, electricista, trabajador industrial de gaseosa, escamador, fotocopiador, gomería, heladería, lavaplatos, limpia lápidas, limpieza, lustra calzados, mecánica, minería, niñera, atención de parqueo vehicular, peinado, repartidor de repostería, sastrería, secretariado, soldador, atención de surtidos de hidrocarburos, taxista, vendedor, trabajos varios. Los sectores más destacados son: comercio, albañilería, mecánica, minería, limpieza, labores de casa y niñera.

Los NAT son un colectivo muy vulnerable y en alto riesgo, porque provienen de familias en extrema pobreza, la mayoría migrantes del área rural con bajo nivel de escolaridad, situadas en el área periurbana de la ciudad de Potosí. Esta situación negativa hace de los NAT que no sean sujetos plenos de derecho; gran parte no asiste a la escuela, muy pocos llegan al bachillerato y solo unos cuantos a la universidad y/o algún instituto de formación profesional; tampoco tienen el cuidado de su salud por parte de sus padres y mucho menos del Estado. La mala alimentación provoca un bajo rendimiento escolar y laboral, que no es comprendido por sus empleadores, los profesores y la misma población que recibe sus servicios.

La situación de inestabilidad socioeconómica en la que viven las familias de los NAT ocasiona que ellos sean sujetos de maltrato físico y psicológico. El maltrato que reciben de sus progenitores estimula a que vean en la calle y en el trabajo una opción para poder alejarse de este ambiente hostil; sin embargo, la calle no es mejor que la casa: muchos NAT enfrentan riesgos como el acoso sexual, la explotación laboral, la 
CHIRI WAYRITA: LA VOZ DE LOS SIN VOZ. UNA EXPERIENCIA DE EDUCACIÓN TRANSFORMADORA PARA LOS NIÑOS Y NIÑAS DEL CERRO RICO (BOLIVIA) M. ${ }^{a}$ DOLORES FERNÁNDEZ MALANDA Y MARA GARCÍA RODRÍGUEZ

delincuencia, diferentes drogodependencias y adicciones, la prostitución y el acoso de las pandillas (García Rodríguez, 2017).

La falta de protección, de afecto, de comunicación intrafamiliar es la causa de traumas psicoemocionales, que les llevan a buscar espacios de apoyo y afecto, como las pandillas en las que se acaban involucrando en actividades delictivas. Además, las escasas posibilidades de acceso a la educación secundaria o a una formación profesional quedan frustradas. Aquellos que logran estudiar tienen que lidiar con la discriminación e intolerancia de las autoridades y docentes, fomentando el abandono escolar (García Rodríguez, 20I5).

Frente a esta realidad, existen instancias del Estado como las Defensorías de la Niñez y la Adolescencia, el Servicio de Gestión Social, la Dirección Departamental del Trabajo y los Juzgados de la Niñez y la Adolescencia encargadas de brindar apoyo, protección y defensa de sus derechos y de su seguridad asignadas por la Ley 2026 (Código Niño, Niña y Adolescente, 20I4), pero lamentablemente no pueden cumplir su rol por la falta de recursos económicos y de profesionales, por lo que existe una seria desprotección de los NAT tanto a nivel estatal como familiar.

\section{La escuela Robertito}

En este contexto, en un país plurinacional, en el que los niños, niñas y adolescentes lograron cambiar una Constitución para que su trabajo fuera reconocido legalmente, en la mina conocida como la Mina del Diablo, asoma una escuelita, pequeña, valiente, a la que asisten un grupo de niños y niñas trabajadores y palliris de las minas.

Cuatrocientas personas viven en el cerro y casi la mitad son niños y niñas, de los cuales menos de la mitad pueden acudir al centro educativo. Junto a la escuela las bocaminas, los caminos inaccesibles entre barro y derrumbes, las grandes máquinas que siguen horadando las entrañas de la montaña. Hierros, pedregales, el frío o el calor abrasador, las detonaciones a escasos metros del edificio escolar cuyas paredes y suelos se abren..., el plomo de los pasivos ambientales que los niños y niñas respiran todo el tiempo...

Constituida conjuntamente por la ONGD Voces Libres y la comunidad del Cerro, de modo especial por las madres que se organizaron para la construcción de las aulas, acoge alrededor de unos 70 niños y niñas de las minas, desde los 2 hasta los I4 años. Allí, seis profesores/as de las Escuelas de Cristo y una pedagoga contratada por Voces Libres dan clases, organizan el almuerzo, y por las tardes ofrecen apoyo escolar a los niños y a sus familias (clases de alfabetización de español siguiendo el sistema de Paulo Freire para algunos padres y madres de las naciones quechuas y aymaras que solo conocen su lengua materna).

Esta modesta escuelita, cuyo objetivo inicial era ofrecer a los niños un acceso integral a la educación, dándoles herramientas para desarrollar sus talentos y salir del ámbito inhumano de la mina, asume también la función de espacio de reuniones comunitarias a través de los diferentes programas de apoyo ofrecidos por ONGD como Voces Libres, Save the Children o PASOCAP (Pastoral Social Cáritas de Potosí que apoya con el programa de «Fortalecimiento y empoderamiento del Movimiento de los NAT») sobre salud, apoyo social y jurídico, talleres de sensibilización contra la violencia de género (mujeres y niños), derechos humanos, el apoyo a microcréditos, 
biblioteca, educación para la higiene, apoyo alimentario y distribución de ropa y zapatos.

La escuela, más allá del abnegado trabajo académico de los profesores, se ha convertido a lo largo de los años en un lugar de expresión y de terapia -pintura, canto, teatro, baile y música- ofrecida por la Universidad Domingo Savio, a través de alguno de los programas del Servicio de Extensión y Responsabilidad Social Universitaria y de las prácticas curriculares de sus estudiantes de Psicopedagogía, ya que «el eterno violento» de la mina hace que los niños y las niñas se encuentren, a menudo, bajo un estado de mutismo y de traumatismo severo.

Resulta difícil para los pequeños habitantes del Cerro Rico seguir un programa escolar normal sin antes trabajar de modo concienzudo y con enorme sutileza la expresión descarnada de sus temores. Los horrores que sacuden el diario de estos niños tienen nombres como derrumbe (las cifras oficiales no lo mencionan pero en el Cerro hay años que mueren hasta 300 personas), desapariciones (trata y tráfico de menores, etc., desaparecen muchos niños y niñas en Bolivia, y en el caso de los niños del Cerro el número es mayor), sacrificios (los ministros del diablo o señores de las minas que hacen sacrificios humanos para que el Señor de la mina les regale una veta... forma parte de la superstición pero lo más probable es que los niños desaparezcan no para los sacrificios sino por temas vinculados al tráfico de órganos), violaciones (de los mineros a las niñas, adolescentes y mujeres del Cerro por el consumo brutal de alcohol), hambre, palizas y abandono.

También es imposible hablar del Cerro Rico, de las minas, de la escuela Robertito y del imaginario de los menores y los adultos, sin mencionar a aquel que «rige» sus destinos: el Tío. El Tío es uno de los dioses menores de la cultura y tradición bolivianas, sincretismo entre el catolicismo y las deidades indígenas. Es el Señor Único de los inframundos, de la oscuridad, del Manqha Pacha o mundo de abajo (Bascopé, 20I3), ofreciendo bajo su gobierno dictatorial protección a los mineros, pero también ruina y destrucción a quienes no le hacen ofrendas en forma de cigarros, hojas de coca o alcohol, y sacrificio de llamas a la entrada de la mina donde esparcen su sangre (Montoya, 2000).

\section{I. La voz de los $\sin v o z$}

De entre todos los proyectos de la escuelita Robertito ha destacado en estos últimos años por la huella dejada Chiri Wayrita (vientito frio en quechua), una propuesta creativa elaborada por los propios niños y niñas del Cerro, y desarrollado por la Escuela de narradores nacida en el seno de la Universidad Privada Domingo Savio (Sede de Potosí) como un colectivo de narración oral que adoptó este nombre para crear un proyecto cultural que transformara las voces de los pequeños en cuentos con los que reivindicar sus derechos a jugar, a opinar y a soñar. El Chiri Wayrita es también el aliento del Cerro Rico que desde la psicomagia se llevó un día las voces de los niños y las niñas que viven en el contexto minero.

Aunque la idea surge en el 2013 por parte del colectivo de proyectos sociales de la UPDS, la propuesta se inició en el 2014 con algunos círculos creativos y espacios de diálogo de la ciudad de Potosí para trabajar la resiliencia y la recuperación del niño interno con los pequeños del Cerro a partir de la narración oral, entendiendo que la 
CHIRI WAYRITA: LA VOZ DE LOS SIN VOZ. UNA EXPERIENCIA DE EDUCACIÓN TRANSFORMADORA PARA LOS NIÑOS Y NIÑAS DEL CERRO RICO (BOLIVIA) M. ${ }^{a}$ DOLORES FERNÁNDEZ MALANDA Y MARA GARCÍA RODRÍGUEZ

vida del Cerro no solo vulnera sus derechos como niños en los aspectos más básicos (salud, educación...), también mutila su creatividad condenándoles a continuar en un «oficio» que pospone indefinidamente su niñez. La propuesta desde su origen ha sido coordinada por Rocío Álvarez Aranibar, técnico de Extensión Universitaria de la UPDS; bajo la dirección de Rosario Puma, vicerrectora de Investigación de la UPDS, y con el apoyo de la Universidad de Burgos a través del Vicerrectorado de Internacionalización y Cooperación.

Durante todos estos años, y una vez a la semana, ocupando toda una mañana, el colectivo Chiri Wayrita de narración oral y escénica ha subido al Cerro, al espacio de la escuela Robertito, con estudiantes de la UPDS y de la UBU para desarrollar actividades vinculadas con la palabra y la escenificación de la misma.

En 2015 se publicó el libro Chiri Wayrita, financiado por la cooperación suiza en Bolivia a través del Fondo para Proyectos Culturales de la Embajada de Suiza en Bolivia y ejecutado por Solidar Suiza, la upDs y el alumnado de la Universidad de Burgos en prácticas curriculares. Más de 20 historias dibujadas por los pequeños de la escuela. Ocho niños/as de nivel inicial en familia comunitaria de primer grado, veinte de nivel inicial en familia comunitaria de segundo grado, diecisiete de nivel primaria comunitario vocacional de primer grado, nueve de segundo grado de primaria comunitaria vocacional, dieciocho de tercer grado de primaria comunitaria vocacional, once de cuarto grado de PCV, doce de quinto grado de PCV y siete de sexto grado de PCV han convertido sus cuentos en un pequeño «dispositivo de transformación social».

Títulos como Los millonarios van al infierno, El lugar donde los abrazos curan, El oro maldecido o el cuento de La niña abandonada hablan de todos esos sueños y miedos que pueblan el día a día de estos niños cuyos héroes son personajes mágicos que les defienden de la maldad de los hombres y del Tío, como la anaconda y el cóndor que «concede poderes para que día y noche protejan a los niños y niñas», como escribe Alexander Pérez Valverde en su cuento Los guardianes del Cerro, o la luna que se casó con el Tío y desde ese día «las personas del Cerro eran más felices porque ya no había muertes por accidentes, así los niños y niñas podían jugar protegidos por la luna», como narra Ever Ticona Mamani en La luna y el Tío.

En noviembre del 2015 se presentó el libro de cuentos y se desarrolló el Primer Festival de Narración Oral Chiri Wayrita en la simbólica Casa de la Moneda, el edificio más emblemático de la ciudad de Potosí, allí donde llegaba la plata arrancada a la montaña y acuñada en monedas que se llevó durante 400 años la Colonia a España. En el evento cultural se reunieron narradores de diferentes partes de Bolivia y de otros países, incluido España, con el colectivo, los educadores de la escuela, algunos padres y los niños y niñas que integran el proyecto, así como autoridades de Potosí. Muchos de los niños que bajaron del Cerro al festival era la primera vez que estaban en la ciudad; ellos y ellas fueron los protagonistas definitivos de un día que jamás olvidarán.

Con los cuentos se logró generar un espacio de horizontalidad, en el que los narradores y educadores de la escuela han diseñado las actividades con los niños, y que permitió trabajar tres componentes esenciales para ayudar a los niños y niñas de Robertito a empoderarse.

La intervención del proyecto Chiri Wayrita, más allá de la parte lúdica que acabamos de referir brevemente, y que resultó esencial para que los niños se abrieran, se ha centrado a lo largo de estos años en:

I. Promoción del protagonismo de los NAT. 
2. Promoción del derecho a la educación y la salud.

3. Recuperación del niño interno.

El objetivo general del proyecto ha sido todo el tiempo la promoción del protagonismo y la participación activa de los niños y niñas de Robertito para la promoción, el cumplimiento y la defensa de sus derechos en el ámbito familiar, educativo, laboral, organizacional y comunitario, a través de la incidencia en el sector público y privado para que sus propuestas sean escuchadas y se conviertan en Políticas Públicas que los beneficien y mejoren su situación. Todo ello, a partir del fortalecimiento y consolidación de la organización como niños, niñas y adolescentes trabajadores (NAT) y de la promoción de la mejora de las condiciones de educación y salud, intentando reducir la deserción y pérdida del año escolar, y el cuidado y prevención de su estado de salud.

Para ello, la Escuela de Narradores ha contado con la presencia continua de técnicos de Pasocap y de Save the Children, organizaciones que llevan años apoyando y asesorando al colectivo de los NAT en Potosí.

Son muchas las acciones desarrolladas, pero podemos destacar varias:

- Elaboración, implementación y evaluación del plan de formación de líderes.

- Ferias, exposiciones sobre los derechos de la niñez trabajadora con la participación de los líderes infantiles.

- Encuentros departamentales y nacionales de los NAT para definir sus líneas base de acción en incidencia política y reivindicación de sus derechos.

- Talleres de capacitación y formación de grupos de base.

- Organización y ejecución de campañas de sensibilización y dignificación del trabajo infantil organizadas por el colectivo para visibilizar su situación.

- Reuniones con autoridades educativas y políticas de la ciudad.

- Apoyos y seguimiento de los NAT en el proceso de permanencia en la escuela.

- Talleres de sensibilización a padres de familia.

- Implementación de becas de estudio que no obligan a dejar el trabajo, sino a establecer turnos de menos horas.

- Elaboración de diagnósticos de salud.

El proyecto es muy amplio y de gran complejidad, y nuestra presencia como profesores y estudiantes de la Universidad de Burgos se centró específicamente en la formación en liderazgo, la gestión de proyectos, el refuerzo escolar y la intervención familiar, todo ello desde la prosocialidad, es decir, en la búsqueda de vías, métodos e instrumentos que hicieran posible la introducción, formación, promoción, mantenimiento e incremento de la prosocialidad en un contexto de Comunidad como es el caso de los habitantes del Cerro.

Para ello presentamos un programa para la optimización de las actitudes y comportamientos prosociales en el marco de la conNAT'sop (Organización que aglutina a todos los NAT del departamento de Potosí), así como propuestas para trabajar el conocimiento y la gestión de las emociones y la autoconciencia, y para ello hicimos uso de taxonomías de comportamientos prosociales basadas en la ayuda y servicio físico, dar y compartir, ayuda y consuelo verbal, valorización y confirmación positiva del otro, escucha profunda y empatía, solidaridad, presencia positiva y unidad, ya que algunos de los profesores participantes en el proyecto han recibido formación con el Dr. Robert Roche en educación prosocial de las emociones. 
CHIRI WAYRITA: LA VOZ DE LOS SIN VOZ. UNA EXPERIENCIA DE EDUCACIÓN TRANSFORMADORA PARA LOS NIÑOS Y NIÑAS DEL CERRO RICO (BOLIVIA) M. ${ }^{a}$ DOLORES FERNÁNDEZ MALANDA Y MARA GARCÍA RODRÍGUEZ

Quizás uno de los talleres más reveladores y que más cambio ha provocado en los niños ha sido la adaptación de los trabajos de John Bradshaw para la reivindicación y recuperación del niño/a interno. A través de dramatizaciones, role-playing, presentación de dilemas morales, dibujos, cuentos, el niño/a se ha enfrentado a su realidad con otros ojos y con el apoyo fundamental del grupo, desde la recuperación y defensa de niño herido.

\section{Conclusiones}

No hay resultados cuantificados. El grupo ha expresado y sigue expresando libremente sentires y emociones. Niños y niñas con vidas difíciles de concebir, con una infancia mutilada en casi todos sus elementos y que no pueden cambiar su presente han descubierto la fuerza del grupo de iguales, se han organizado, y aunque no han salido de su espiral de pobreza van recuperando poco a poco su voz... no todos. Algunos y algunas permanecen en el mutismo. No podemos impedir que les exploten, les maltraten y les abusen. El sueño de los profesores de Robertito es que un día no haya ningún niño trabajando en el Cerro y que la escuela se cierre porque ya no queden niños en la montaña del diablo. Mientras, ellos y ellas, con el apoyo de personas que sueñan otro mundo para la infancia, seguirán dando sus clases, sin sufrir como los maestros de otras escuelas de parálisis metodológica, porque más allá de los contenidos necesarios entienden la necesidad de fortalecer en un medio tan hostil el sentimiento de concordia y la correspondencia con el otro, la prosocialidad y la resiliencia.

\section{Bibliografía}

AA. vv. (20I5). Chiri Wayrita. Cuenta cuentos. La Paz: Instituto Internacional de Integración del Convenio Andrés Bello.

Asamblea Constituyente de Bolivia. Congreso Nacional. (2009). Constitución Política del Estado Plurinacional de Bolivia.

Banchón, M. (2015). Bolivia defiende en Bruselas su ley de trabajo infantil. Recuperado de: http://dw.com/p/rEPDv.

Battle, R.; Puig, J.; Bosch, C. y Palos, J. (2007). Aprendizaje Servicio. Educar para la ciudadanía. Barcelona: Octaedro.

Bascopé, V. (2013). Espiritualidad originaria en el Pacha Andino. Aproximaciones teológicas. Cochabamba: Editorial Verbo divino, $3 .^{\text {a }}$ edición.

Campos, R.; Citarella, L. y Zangari, A. (20I0). Yachay Tinkuy. Salud e intercultural en Bolivia y América Latina. La Paz: Editorial Gente Común.

Censo del Instituto Nacional de Estadística de Bolivia. (20i2). Bolivia. Disponible en http://www.ine.gob.bo/.

CEPAL. (20I8). Estudio económico de América Latina y del Caribe. Evolución de la inversión en América Latina y el Caribe: hechos estilizados, determinantes y desafíos de politica. Disponible en https://www.cepal.org/es/publicaciones/43964-estudio-economico-america-latina-caribe-2or8-evolucion-la-inversion-america.

Comisión Europea. Dirección General de Educación y Cultura. (2009). Marco de Cualificaciones Europeas para el aprendizaje a lo Largo de la Vida EQF-MEC. Luxemburgo: Oficina de Publicaciones Oficiales de las Comunidades Europeas. 
Fernández Malanda, D. (20i6). El 'Sexto Sol' o el mito de la esperanza del retorno de los tiempos nuevos: breve aproximación a la espiritualidad del Pacha Andino. En E. Gómez Campelo y E. Cifuentes García (coords.) Nuevas concepciones sobre el desarrollo en América Latina: elementos para el debate desde los movimientos sociales y la Universidad. Burgos: Ediciones Universidad de Burgos.

García Rodríguez, M. (20I5a). Hacia el empoderamiento emocional de los adolescentes trabajadores del Centro minero "Yachay Mosoj». Programa de Educación Afectivo sexual (20102013). Tesis inédita. Universidad de Burgos.

García Rodríguez, M. (20i5b). Percepciones de los adolescentes trabajadores bolivianos sobre violencia física y psicológica mediante la técnica de grupos de discusión. Revista Cientifico-Técnica sobre la Infancia y Adolescencia (REINAT).

García Rodríguez, M. (20I7). Peores formas de trabajo infantil: la realidad que desafía a la Ley (pp. I083-IIO2). Lozano Impresores, s.L. Ed. GEU.

LAtorre, A. (2003). La investigación-acción: Conocer y cambiar la práctica educativa. Barcelona: Graó. Capítulo 2.

Montoya, V. (2000). Cuentos de la Mina. Editorial Norte. Colección las Voces de la Tierra. pasocap (20I4). Proyecto "más allá del sol». Potosí, Bolivia: [s. n.].

PASOCAP (20I5). Proyecto «Promoviendo el protagonismo y la participación de los NAT's para la defensa y cumplimiento de sus derechos». Potosí, Bolivia: [s. n.].

www.gacetaoficialdebolivia.gob.bo. Ley 70. Ley de educación «Avelino Siñani Eliardo Pérez», 20 de diciembre de 2010. 